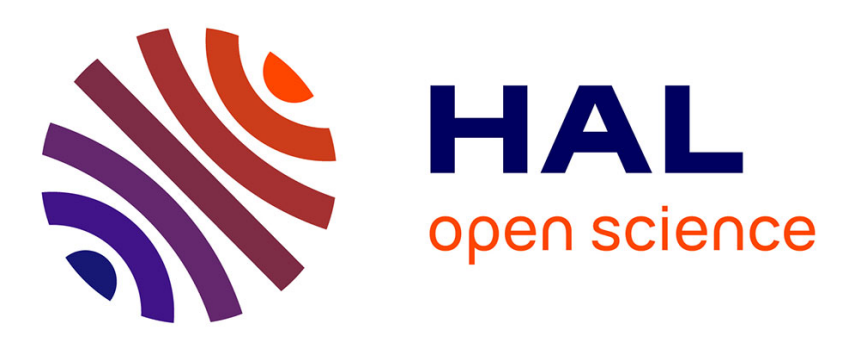

\title{
LiFi Experiments in a Hospital
}

Sreelal Maravanchery, Peter Hellwig, Jonas Hilt, Kai Lennert Bober, Volker Jungnickel, Klara Hirmanova, Petr Chvojka, Radek Janca, Stanislav Zvanovec

\section{To cite this version:}

Sreelal Maravanchery, Peter Hellwig, Jonas Hilt, Kai Lennert Bober, Volker Jungnickel, et al.. LiFi Experiments in a Hospital. Optical Fiber Communication Conference (OFC) 2020, Mar 2020, San Diego, United States. pp.M3I.2, 10.1364/OFC.2020.M3I.2 . hal-02569803

\section{HAL Id: hal-02569803 https://hal.science/hal-02569803}

Submitted on 11 May 2020

HAL is a multi-disciplinary open access archive for the deposit and dissemination of scientific research documents, whether they are published or not. The documents may come from teaching and research institutions in France or abroad, or from public or private research centers.
L'archive ouverte pluridisciplinaire HAL, est destinée au dépôt et à la diffusion de documents scientifiques de niveau recherche, publiés ou non, émanant des établissements d'enseignement et de recherche français ou étrangers, des laboratoires publics ou privés. 


\title{
LiFi Experiments in a Hospital
}

\author{
Sreelal Maravanchery Mana ${ }^{1}$, Peter Hellwig ${ }^{1}$, Jonas Hilt ${ }^{1}$, Kai Lennert Bober ${ }^{1}$, Volker Jungnickel ${ }^{1}$ \\ Klara Hirmanova ${ }^{2}$, Petr Chvojka ${ }^{3}$, Radek Janca ${ }^{3}$, Stanislav Zvanovec ${ }^{3}$ \\ ${ }^{1}$ Fraunhofer Institute for Telecommunications, Heinrich-Hertz Institute (HHI), Einsteinufer 37, 10587 Berlin, Germany \\ ${ }^{2}$ Department of Medical Technology, Motol University Hospital, Prague 150 06, Czech Republic \\ ${ }^{3}$ Faculty of Electrical Engineering, Czech Technical University in Prague, Prague 16627, Czech Republic \\ sreelal.maravanchery@hhi.fraunhofer.de
}

\begin{abstract}
We present LiFi channel measurements in a neurosurgery room of Motol University Hospital in Prague. Individual channels are combined into a virtual multiuser MIMO link. We report achievable data rates for different $\mathrm{LiFi}$ transmission schemes.

OCIS codes: (060.0060) Fiber optics and optical communications, (060.2605) Free-space optical communication
\end{abstract}

\section{Introduction}

The connected or smart hospital is widely considered as the future of healthcare, where all medical devices are connected to a wireless infrastructure and provide accurate and timely information [1-4]. Aiming at safe and secure wireless communication, the use of wireless local area networks such as WiFi in surgery environments is limited due to possible electromagnetic interference (EMI). Hence, the deployment of a networked optical wireless technology ( $\mathrm{LiFi}$ ), based on the light has the potential to be employed in hospital environments [2]. Recently, several research groups provided insights into the potential of LiFi in healthcare environments [1-3]. For introducing $\mathrm{LiFi}$ in hospitals, engineers need a fundamental understanding of the propagation scenario, i.e. the impact of blockages and reflections by objects. Accordingly, robust transmission schemes need to be developed to enable reliable wireless communication and high data rates. In this paper, we report for the first time on an experimental investigation of LiFi transmission in a typical neurosurgery room. We recorded frequency responses of the optical channels for various transmitter (Tx) and receiver (Rx) positions in the room and considered them subsequently as a virtual multiuser multiple-input multiple-output (MU-MIMO) system. Finally, the achievable data throughputs for possible LiFi transmission schemes are estimated.

\section{Measurement Scenario}

Fig. 1 shows the measurement scenario. Experiments were performed in the children neurosurgery room of Motol University Hospital in Prague, Czech Republic. Fig. 1(a) shows the photo of the operating room, the positions of Rx and Tx are highlighted in Figs. 1(b) and (c), respectively. The existing communication systems in this environment are: $i$ ) body sensor network (BSN); ii) infrared (IR) navigation for precise position detection; iii) high-resolution cameras for monitoring and recording the surgery and $i v$ ) endoscopes. Here, we investigate the performance of a LiFi system employed in the operating room for downlink transmission of BSN. Tx and Rx are each fixed at six different positions. All Rxs are placed on the operating table with $1 \mathrm{~m}$ height looking upwards to the ceiling where the Txs are mounted at around $2 \mathrm{~m}$ height with a certain tilt looking downwards to the operating table. Multiple Rxs represent multiple sensors, while multiple Txs are used in order to avoid possible blockage of the line-of-sight (LOS) due to devices and humans moving inside the room.

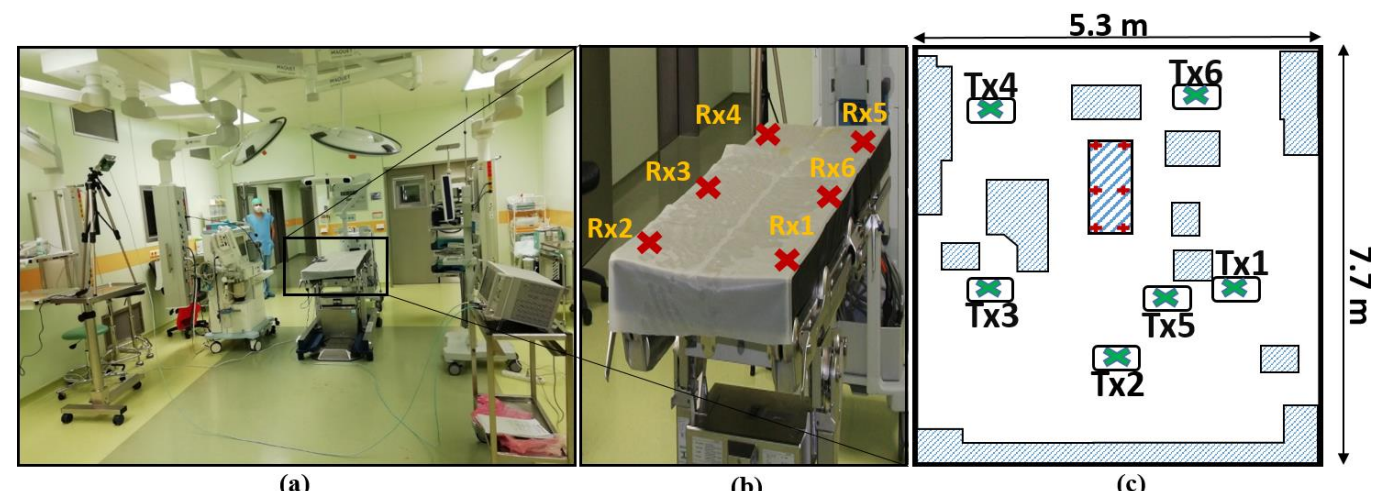

(a)

(b)

(c)

Fig.1: Measurement Scenario: (a) Neurosurgical operating room; (b) Positions of Rx in the operating table; (c) Top view of the surgery room where the Tx positions are marked in green color. Here Rx positions are marked in red 
All measurements are conducted in the same static environment one after the other for each Tx-Rx link. Here, the conventional frequency sweep technique is adopted [5]. A standard Agilent vector network analyzer (VNA E5061B3L5) is connected between Tx and Rx feeding a continuous wave signal with variable frequency from 1 to $200 \mathrm{MHz}$. For characterizing each channel, the optical frontend response is calibrated out, which is measured at a minimum distortion-free distance in free LOS [6]. All the channel responses and channel parameters are calculated as given in [6].

\section{Channel Characterization}

From the experimental data, it is observed that the signal strength is weaker with respect to all $\mathrm{Rx}$ when using Tx 1 and $\mathrm{Tx} 2 . \mathrm{Tx} 1$ is often blocked by a monitor while $\mathrm{Tx} 2$ is too far away from the operating table. Here, we report only channel characteristics of the other four Tx positions with respect to each Rx position. The measured magnitude responses of the channels are shown in Fig. 2(a).

$\mathrm{Rx} 1$ and $\mathrm{Rx} 2$ are placed at the head position of the patient which has good visibility and free LOS from Tx5 and Tx3. Similarly, Rx4 and Rx5 are nearby the leg position of the patient, where Tx4 and Tx6 have good signal strength. The amplitude responses for Rx3 and Rx6 are always between the maximum and minimum response, since they are located at the center position of the operating table. The initial drop at the lower frequencies of the amplitude response indicates that there are many diffuse reflections in the surgery room where all objects have bright colors (see Fig. 1(a)). Flatness at higher frequencies is due to the presence of the LOS.

\section{MU-MIMO scenario}

In our architecture, we assume that all transmitters are coordinated from a central unit which enables simultaneous transmissions from multiple transmitters, i.e. spatial diversity to prevent sudden blockages of the LOS. Moreover, joint scheduling of multiple user transmissions is possible in the space and time domains. The MU-MIMO link is commonly characterized by using the singular values of the compound channel matrix $H$, which contains all the links between all Txs and all Rxs. The singular values of $H$ are plotted in Fig. 2(b) which shows that 2 of them are much stronger than the two others. This suggest that at least 2 data streams can be spatially multiplexed, i.e. transmitted in parallel, in our specific scenario. Figs. 2(c) shows the normalized channel gains at $1 \mathrm{MHz}$ between all Txs and all Rxs, and indicates that each Rx has at least 2 strong Tx links. Fig. 2(d) confirms our expectation that the delay spread is low if the path gain is high and vice versa. From our observations, it is intuitive that Tx 3 and Tx5 send a first data stream to Rx1 or Rx2. At the same time, Tx4 and Tx6 send a second data stream to Rx3, Rx4, Rx5 or Rx6. In this way, each Rx has data signals coming from two Txs and potential interference from two other Txs. This ensures reliable transmission as one LOS could be blocked while the transmission is ongoing.

\section{MU-MIMO results}

Now we assume three possible transmission schemes and evaluate the achievable throughput. By assuming equal transmit power, the signal to interference noise ratio (SINR) is calculated for each Rx by using the magnitude response of the channel. For estimation of SINR, however, noise power is unknown. The maximum SNR that can be achieved using our frontend design is $45 \mathrm{~dB}$. Hence, we assumed a constant noise floor at a level which is $45 \mathrm{~dB}$

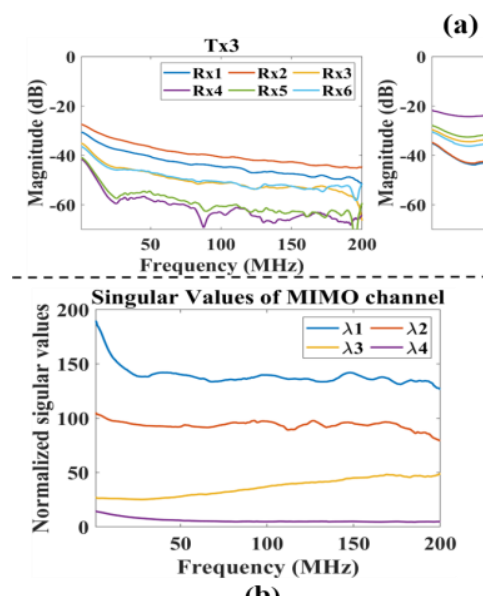

(b) (a) Magnitude response of the $\underset{T \times 4}{\operatorname{Tx} 5}$
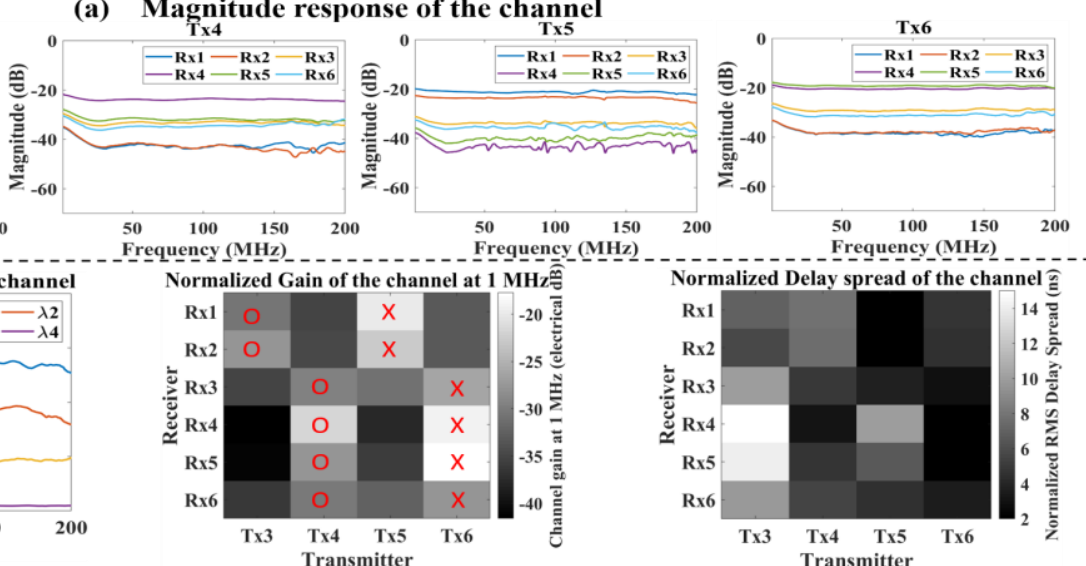

(c)

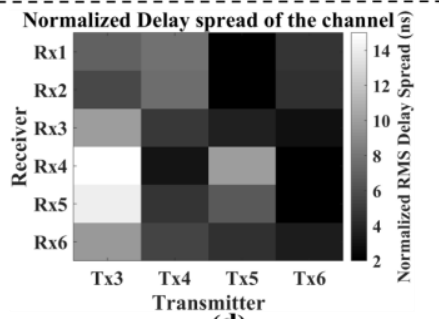

(d)

Fig. 2: Results: (a) Magnitude response of channels for each Tx; (b) Singular values of 4x6 distributed MIMO; (c) Normalized gain at 1 MHz. Here first maxima indicated as X and second maxima as O; (d) Normalized delay spread of the channel 

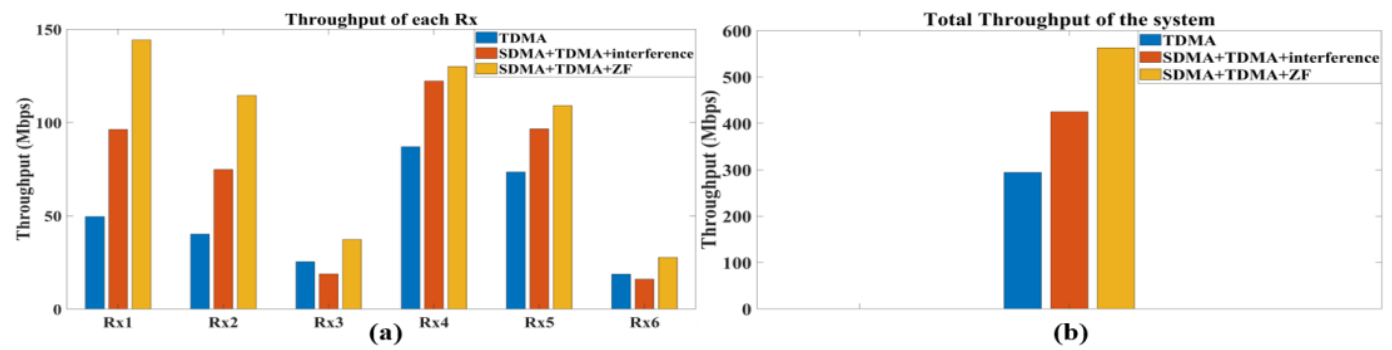

Fig. 3 Results: (a) Throughput of each Rx at $10 \mathrm{MHz}$; (b) Total throughput of the system at different transmission schemes at $10 \mathrm{MHz}$

below the maximum magnitude response of the LiFi system measured at the minimum distortion-less distance. From the estimated SINR, the throughput TP of each individual Rx is calculated by means of a modified Shannon formula $T P=B^{*} \log _{2}(1+\mathrm{SINR} / \Gamma)$ where $B$ is the bandwidth and $\Gamma=10$ is assumed taking imperfect constellation shaping, limited code word length and limited modulation index to avoid clipping into account. Note that the above formula is applied on each frequency and then summation is applied over the entire bandwidth, i.e. $200 \mathrm{MHz}$.

The first scheme is classical time-division multiple access (TDMA) where each user is assigned one out of six exclusive time slots and served by its best two Txs. In the second scheme, TDMA is extended by space division multiple access (SDMA) where half of the time in a particular time slot is scheduled for Rx1 and Rx2 in order to receive first data stream from Tx 3 and Tx 5 and other Rx has simultaneously one by fourth of the time to receive the signals from Tx4 and Tx6. Thus, an interference term is included in the SINR calculation depending on the selected Txs and Rx. Finally, the zero forcing (ZF) method has been applied after the same scheduling used in the TDMASDMA approach. Note that the effective MIMO channel gain matrix in each time slot is given as $2 \times 2$ matrix. A Moore-Penrose pseudo-inverse of the channel gain matrix is calculated as in [7]. All elements of the inverse matrix are normalized with respect to the row norm to avoid the power fluctuations and send with equal power to each user. Here the ZF is computed in each time slot and it executes the channel inversion for removing the interference. Note that, $\mathrm{Rx} 1$ and $\mathrm{Rx} 2$ have higher throughput since they are available for longer time with stronger signals.

Fig. 3 shows the estimated throughput of the investigated configurations. From Fig. 3(a) it is clear that the ZF method brings a significant improvement in the throughput for each Rx. For Rx3 and Rx6, TDMA method is much better than SDMA and TDMA due to high interference from the other sources. The total throughput of the system is shown in Fig. 3(b). We can observe that the throughput of the system with ZF is around two times higher than TDMA alone and 1.5 times higher compared to the interference limited case.

\section{Conclusion}

In this paper, we report the first experimental study of LiFi system in a medical scenario. The frequency response of the LiFi channels were measured in a neurosurgical operating room and corresponding channel parameters were calculated. By considering all measured channels as a virtual distributed MU-MIMO system, multiplexing schemes such as i) TDMA; ii) TDMA-SDMA and iii) TDMA-SDMA with ZF were considered and the achievable throughputs were estimated. We observed that TDMA-SDMA with ZF provides the highest possible throughput.

\section{Acknowledgement}

The authors acknowledge the Department of Neurosurgery, $2^{\text {nd }}$ Faculty of Medicine, Charles University in Prague and Motol University Hospital, Prague, Czech Republic for all the provided help during experiments. This research is funded by the VisIoN, a European Union's H-2020 MSCA ITN program under the grant agreement no 764461.

\section{References}

[1] Y.K. Cheong et al., "Hazardless biomedical sensing data transmission using VLC," IEEE Sensors J., vol. 13, no. 9,pp. 3347-3348, Sep. 2013.

[2] L. Chevalier et al., "Optical Wireless Links as an Alternative to Radio-Frequency for Medical Body Area Networks," JSAC, vol.33, no.9, pp.2002-2010, Sept. 2015.

[3] S. S. Torkestani et al., "Indoor optical wireless system dedicated to healthcare application in a hospital," IET Commun., vol. 6, no. 5, pp. 541547, Mar. 27, 2012.

[4] R. Murai et al., "A novel visible light communication system for enhanced control of autonomous delivery robots in a hospital," SII, IEEE/SICE International Symposium on, pp. 510-516, Fukuoka, 16-18 Dec 2012.

[5] J. M. Kahn et al., "Experimental characterization of non-directed indoor infrared channels," in IEEE Transactions on Communications, vol. 43, no. 2/3/4, pp. 1613-1623, 1995.

[6] S. M. Mana et al., "Experiments in Non-Line-of-Sight Li-Fi Channels," 2019 Global LIFI Congress (GLC), Paris, France, 2019, pp. 1-6.

[7] Q. H. Spencer et al., "Zero-forcing methods for downlink spatial multiplexing in multiuser MIMO channels," IEEE Trans. Signal Process., vol. 52, no. 2, pp. 461-471, 2004. 